\title{
ON THE RELATIVITY OF PERCEIVED MOTION
}

\author{
A.H. WERTHEIM * \\ Institute for Perception TNO, The Netherlands
}

\begin{abstract}
Perceived stability of the visual world during eye movements is traditionally explained as due to the presence of extraretinal signals, equal in magnitude to retinal signals. Motion is perceived when the two signals differ. An experiment is reported in which motion thresholds were measured during smooth pursuit eye movements. The results show that the traditional view is incomplete. Motion is only perceived when the two signals differ by at least a just noticeable difference (JND), the magnitude of which depends on ocular velocity and is independent of the direction of stimulus motion relative to the eyes. The data lead to the rejection of theories according to which ocular velocity is under-represented in extraretinal signals. In addition they show that retinal image motion carries no information about stimulus motion. Perceived motion, direction and velocity are relative concepts. They depend on the JND and therefore they are relative to extraretinal signals. This principle explains the Filehne illusion and even predicts the Aubert-Fleischl phenomenon. A similar analysis can be applied to understand vestibular effects on motion perception.
\end{abstract}

\section{Introduction}

When an eye movement is made over a stationary visual field, the image of that field shifts over the retina. If the eye movement is the result of the intention to move the eyes (and is not induced or affected by the application of an external force to the eyeball) motion of the visual field is not perceived. Apparently, the visual system 'knows' that the image shift was due to the (intended) eye movement. Such knowledge cannot be obtained from what happens on the retina itself. The logical necessity that an extraretinal signal, carrying such information (sometimes termed a 'corollary discharge' or an 'efference copy') is needed for the establishment of a perceptually stable world during eye movements, was recognized long ago (see for some discussions Helmholz 1962; Holst 1954; Gyr 1972; Jeannerod et al. 1979). Nevertheless, the precise nature and function of such extraretinal signals remain controversial because of some anomalous experimental findings.

First, the visual world is not always perceived as stationary during eye movements. When a stationary stimulus is briefly presented during a saccadic

* Author's address: A.H. Wertheim, Institute for Perception TNO, P.O. Box 23, 3769 ZG Soesterberg, The Netherlands.

0001-6918/81/0000-0000/\$02.50 ㅇ 1981 North-Holland 
or smooth pursuit eye movement in an otherwise dark visual field (e.g. a light flash), an illusory displacement of the stimulus is perceived (e.g. Hazelhoff and Wiersma 1925; Matin 1972; Mohanan 1972; Findlay 1974; Ward 1976; Mateeff 1978). The phenomenon can be explained by the hypothesis that extraretinal signals do not serve to establish a perceptually stable world but instead serve to gather evidence for motion, given an a priori subjective assumption of a stable world (MacKay 1972, 1973; MacKay and Mittelstaedt 1974). According to this view extraretinal information about oculomotor activity is used to form some kind of expectation as to how retinal images would move as the result of eye movements. When this expectation differs from the actual retinal image shift, motion is perceived. The illusory perception of stimulus displacement can be explained by assuming that an eye movement implies the presence of an extraretinal signal proportional to its size. Thus a corresponding retinal image shift is expected. However, this expectation is not born out because a stimulus briefly flashed on during an eye movement (in dark surroundings) creates only a very small retinal image shift. Therefore the stimulus is seen to move [1].

Although this explanation is quite attractive it cannot be applied to another observation: during smooth pursuit eye movements, illusory motion of a stationary stimulus is sometimes perceived, even if the stationary stimulus is continuously present (Filehne 1922; Mack and Herman 1973, 1978).

To explain this finding another hypothesis has been formulated, which states that oculomotor activity is slightly under-registered by the visual system, and thus is under-represented in the extraretinal signal. Therefore, the expected retinal image shift always falls short of the observed one (Mack and Herman 1972, 1973, 1978). Hence all instances of illusory motion during eye movements can be explained.

The underregistration hypothesis gains support from the so-called AubertFleischl phenomenon (Aubert 1886, 1887; Fleischl 1882; Dichgans et al. 1969; Mack and Herman 1972, 1973; Dichgans et al. 1975), which refers to the fact that the perceived velocity of a stimulus pursued with the eyes is lower than when the stimulus moves (with the same velocity) and the eyes are kept stationary.

In the latter case stimulus velocity can only be estimated from a retinal motion signal because the extraretinal signal approximates zero. Conversely, during ocular pursuit of the stimulus, there is almost no retinal signal and thus estimates of stimulus velocity must be derived mainly from oculomotor information carried by the extraretinal signal. Since in this case stimulus velocity is

[1] In the absence of enough time over which the stimulus can be perceived, the impression of motion probably remains fractionary and may become manifest only as a displacement (see Legge and Campbell 1981). 
estimated to be lower, oculomotor information seems to be somewhat underrepresented in the extraretinal signal [2].

A problem with the under-registration hypothesis is that during eye movements an actual movement or displacement of a stimulus is often not perceived: thresholds for motion and displacement are elevated during saccades (Beeler 1967; Mack 1970; Bridgeman et al. 1973; Whipple and Wallach 1978; Bridgeman and Stark 1979; Bridgeman et al. 1979). When stimulus movement is small and is in the same direction as the eye movement this poses no problem for the under-registration hypothesis. In such a case the retinal motion signal is slightly reduced and might become equal to the extraretinal signal, yielding a perception of a stationary stimulus (which implies an increased threshold for motion). But when a stimulus moves in the direction opposite to the eyes, the retinal signal is always larger than the extraretinal signal and thus motion should always be perceived. Nevertheless the increased threshold for motion is observed irrespective of the direction of stimulus motion relative to the eyes (Mack 1970; Whipple and Wallach 1978; Bridgeman and Stark 1979). To complicate matters even more, the threshold increases in direct proportion to the size of the saccade (Mack 1970; Bridgeman et al. 1973).

Presently, there is no theory that can explain all these data taken together, apart from the general statement that extraretinal information is of a "sloppy" nature (Matin 1974; Beeler 1967) or has "disadvantageous side effects" (Büchele et al. 1980). It is the purpose of this paper to provide a better alternative.

Let us assume that oculomotor activity is not under-registered by the visual system. The magnitude of an extraretinal signal can then be thought of as equal to the magnitude of the eye movement with which it is associated. When a stimulus moves slightly in front of the eyes during a normal eye movement, the retinal signal will be either smaller or larger than the extraretinal signal, depending on whether the stimulus moves in the same direction or opposite to the eyes. It is now proposed that, even though in such cases there is a difference between the retinal and extraretinal signals, motion of the stimulus will not be perceived unless this difference reaches a just noticeable magnitude. In psychophysical terms a just noticeable difference (JND) between the two signals must be reached before motion can be perceived.

When comparing two signals, a JND usually becomes larger with increasing magnitude of the reference signal against which the critical signal is evaluated. If this reasoning applies to the visual system, the elevation of motion thresholds

[2] An alternative explanation of the Aubert-Fleischl phenomenon is to assume that for some reason the velocity of retinal image shifts is always overestimated (Dichgans and Jung 1969). Such a "retinal overestimation hypothesis" is not essentially different from the under-registration hypothesis because both imply that extraretinal signals are always somewhat smaller than the concurrent retinal signals. 
during saccadic eye movements can be explained: When a stimulus moves past stationary eyes, the extraretinal signal approximates zero, but the retinal signal has a certain magnitude. In this case the JND approximates the magnitude of the retinal image shift evoked by the stimulus movement at the absolute threshold for the detection of motion. When the eyes move, the extraretinal signal increases. It becomes larger with larger eye movements. Accordingly, the JND increases. Therefore with larger eye movements the movement of the stimulus must be larger if its motion is to be perceived. Hence, the threshold for motion is elevated during eye movements in direct proportion to the magnitude of the eye movement.

The JND may be reached because retinal signals are either sufficiently larger or smaller than the extraretinal signal (dependent on the direction of stimulus motion relative to the eye movement). Therefore the threshold elevation occurs irrespective of whether the stimulus moves against or with the eyes.

As mentioned before, these effects have all been observed during saccadic eye movements, but not with smooth pursuit eye movements. Contrary to saccades, where the eyes do not track a moving stimulus, the characteristics of smooth pursuit eye movements seem to depend on stimulus velocity rather than on the amplitude of the stimulus movement (see Miles and Evarts 1979; Wertheim 1980). Therefore it is reasonable to assume that during smooth pursuit extraretinal signals are coded in terms of the velocity rather than the size of eye movements. According to the present theory the detection of motion then depends on whether the difference between these retinal and extraretinal velocity signals reaches a just noticeable level. The theory thus predicts that, when the eyes track a moving stimulus, the threshold for the detection of motion of another stimulus in the visual field should increase in direct proportion to the velocity of the eyes, irrespective of whether the latter stimulus moves with or against the eyes. In the following experiment this prediction is tested.

\section{The experiment}

\section{Apparatus}

Fig. 1 illustrates the experimental set-up. With the help of a slide projector and a mirror mounted on a galvanometer (A) a visual stimulus, consisting of a patterned visual field (henceforward called background) was projected on a large screen $350 \mathrm{~cm}$ in front of the $S$ 's eyes. The pattern consisted of 240 identical white disks $\left(1.2^{\circ}\right.$ diameter; luminance $2 \mathrm{~cd} / \mathrm{m}^{2}$ ) distributed pseudo-randomly to create an impression of a homogeneously structured rectangular visual field $\left(38^{\circ} \times 20^{\circ}\right)$. The whole background pattern could be moved horizontally to the right or to the left by rotating the mirror.

Another slide projector, mirror and galvanometer (B) projected a target disk, identical to the background disks but brighter $\left(16 \mathrm{~cd} / \mathrm{m}^{2}\right)$. The target could also be moved horizontally to the right or left. The voltages serving as an input for both 


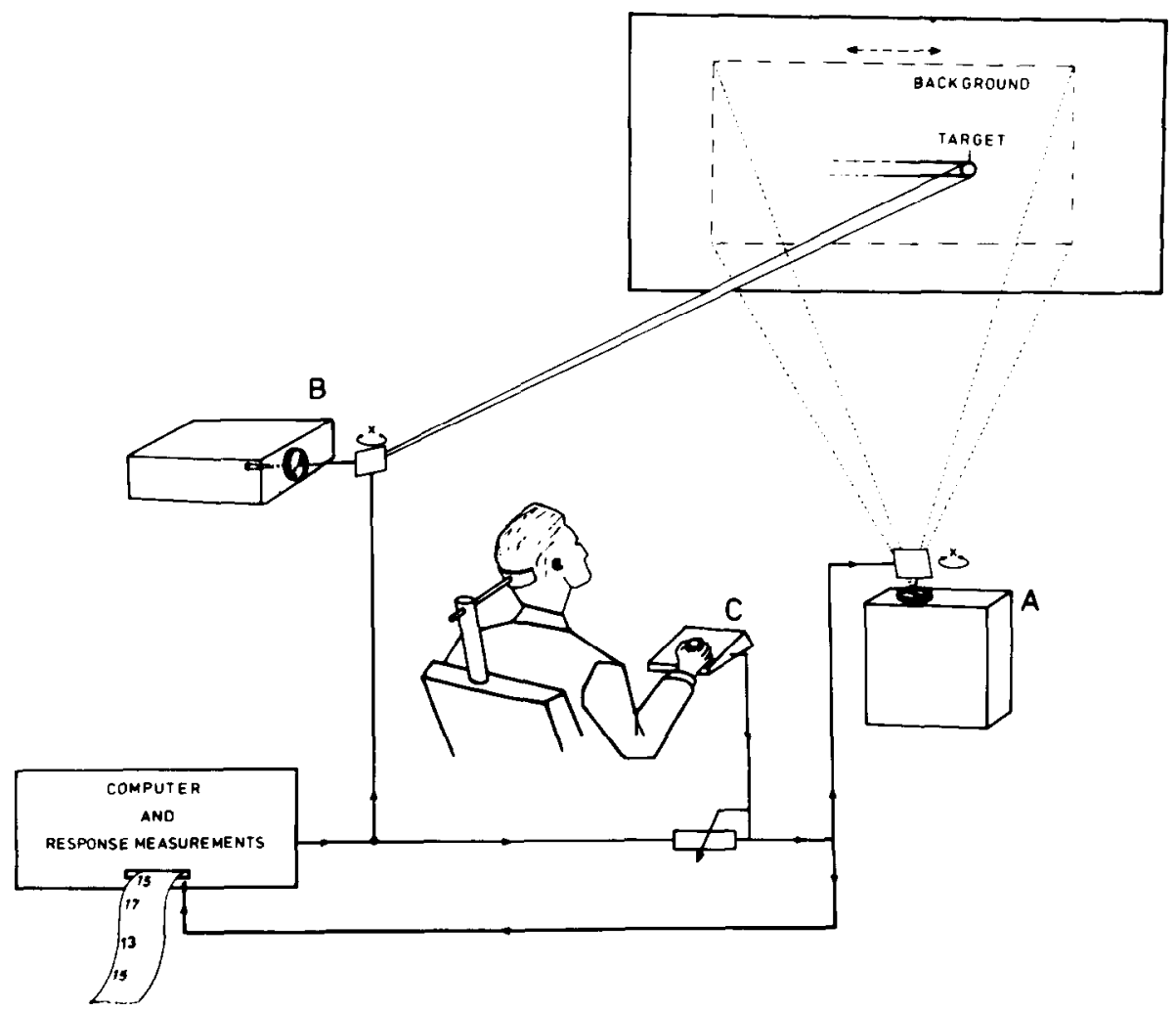

Fig. 1. Experimental set-up.

galvanometers were identical, so background and target always moved in precise temporal synchrony. The voltage was generated and varied by a PDP 11-34 computer. $S$ s could attenuate the input for the galvanometer in A with the help of an adjustable potentiometer (C). In this way they could change the amplitude of the background motion and by doing so they adjusted the velocity of the background motion. The computer measured the voltage of the attenuated signal at the end of each target sweep. This voltage was printed out when the computer received a request pulse from a push button operated by the $S$ (not shown in fig. 1).

From this voltage the velocity (and amplitude) of the background motion could be calculated. By reversing the sign of the input to the galvanometer in $A$ the experimenter could change the direction of movement of the background relative to the target and could make it either opposite or equal to the direction of the target motion.

Target and background moved to and fro with constant velocities. These movements were not strictly triangular, however. In such cases $S$ s usually perceive a jerky kind of motion at the turning points of the target sweeps, due to overshoot of the eyes. Therefore the signals sent to the galvanometers were slightly damped at the turning points. (In a pilot study the degree of damping was determined as the point where this jerky motion was no longer perceived.) 
When a signal reverses its direction each time immediately at the end of a sweep of a given amplitude, a different velocity implies a different frequency. This confounding should be avoided because of possible intervening effects of eye movement frequency (see Wertheim 1979). Therefore the target (and thus also the background) was kept stationary for a certain period (ranging between 2 and $6 \mathrm{sec}$ ) at the end of each sweep. The duration of this rest period was such that the total duration of a sweep plus rest was the same for all conditions. This procedure allowed independent variation of the velocity and the amplitude of target motion, unconfounded with frequency.

\section{Method}

$S$ s were seated in a dimly lit experimental room and maintained their head in fixed position against a head rest attached to the chair. Prior to each trial the potentiometer was set at $100 \%$ attenuation, which means that the background was stationary. The target, which served no other purpose than to induce smooth pursuit eye movements of a certain amplitude and velocity, was then set in motion. The $S$ tracked it with his eyes and at the same time slowly turned the knob of the potentiometer. Hence the background started to move (in temporal synchrony with the target) with an increasing amplitude and therefore with an increasing velocity. The $S$ continued to turn the knob up to the point where he noticed the background motion for the first time. He then turned the knob of the potentiometer slightly back, to the point where the background seemed to be stationary again after which he pressed the button for a request pulse to the computer, which then typed out the voltages from which the velocity (and also the amplitude) of the background motion could be calculated. These measures were taken as the threshold values for the perception of background motion.

The amplitude and velocity of the eye movements were varied independently by varying the amplitude and velocity of the target motion. Four amplitudes (7.5, 15.6, 24.1 and $\left.32.7^{\circ}\right)$ and four velocities $\left(6.5,9.8,13.1\right.$ and $16.4^{\circ} / \mathrm{sec}$ ) were used. These target velocities were chosen because they are well within the range where visual pursuit is highly accurate and where the velocity of the eyes equals the velocity of the target (Dichgans and Jung 1969).

Target and background moved either in the same or in opposite directions. Hence there were a total of 32 experimental conditions, differing with respect to the four target movement amplitudes, the four target movement velocities and the two directions of background motion relative to the eyes. For each $S$ all conditions were replicated three times. The direction of background motion relative to the eyes was varied in two blocks presented in balanced order between $S \mathrm{~s}$. All other conditions were randomized within these two blocks. Measurements were taken in 10-min sessions alternating with 10-min rest periods. 18 male and female students of the University of Utrecht, aged between 18 and 27 years, served as naive $S$ s.

\section{Results}

Mean velocity of background motion at threshold was calculated within each condition for all $S$ s. These scores served as entries in ANOVA with eye movement velocity, eye movement amplitude and eye movement direction relative to the background, serving as three independent factors. As fig. 2 shows, the velocity of background motion at 
threshold increased linearly with eye movement velocity $(F=45.7 ; d f=3,51 ; p<0.001$; $16 \%$ variance explained. The linear regression lines are $y=0.12 x+0.25\left(r^{2}=0.98\right)$ when the background moves against the eyes and $y=0.07 x+0.79\left(r^{2}=0.98\right)$ when it moves with the eyes). There was no main effect of the direction of background motion relative to the eyes $(F<1)$ although it influenced the effect of eye movement velocity slightly $(F=10.3 ; d f=3,51 ; p<0.001 ; 0.9 \%$ variance explained). Neither was there a main effect of eye movement amplitude $(F=1.98 ; d f=3,51 ; p>0.10)$ although this factor also slightly affected the effect of eye movement velocity $(F=2.58 ; d f=9,153$; $p<0.01 ; 0.13 \%$ variance explained). A very small second-order interaction between the effects of all three factors was also observed $(F=2.36 ; d f=9,153 ; p<0.02 ; 0.15 \%$ variance explained).

A similar ANOVA was performed on the mean amplitudes of background motion at threshold. Since amplitude and velocity of background motion were confounded in the experimental design ( $S$ s changed the background velocity by adjusting its amplitude) the results were similar. The only difference was that eye movement amplitude here showed a significant effect. However, this is an artefact: lengthening a target sweep without changing its velocity makes it last longer and the concurrent background motion then also lasts longer (background and target moved in temporal synchrony), but the velocity of background motion remains unchanged (eye movement amplitude does not affect background movement velocity at threshold, see the former ANOVA). A background movement which lasts longer without a change in velocity must have an increased amplitude. The effect of eye movement amplitude on background motion amplitude thus only reflects the temporal synchrony of target and background movements.

The effect of eye movement velocity on background velocity at threshold can more easily be illustrated when this background velocity is added to or subtracted from eye movement velocity (dependent on the directional condition). This yields the velocity of the background image shift over the retina. In fig. 3 this retinal velocity signal is plotted against eye movement velocity. Since it was assumed that the extraretinal velocity signal equals the velocity of the eyes, this graph shows how much the retinal and extraretinal velocity signals must differ before motion of the background can be perceived in both directional conditions. The broken line in fig. 3 (inclined at $45^{\circ}$ angle) shows where the retinal and extraretinal velocity signals are equal.

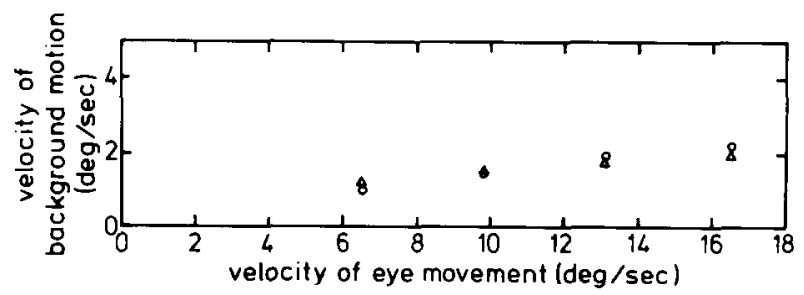

Fig. 2. Velocity of background motion at threshold, as a function of eye movement velocity. Circles: background motion against the eyes. Triangles: background motion with the eyes. 


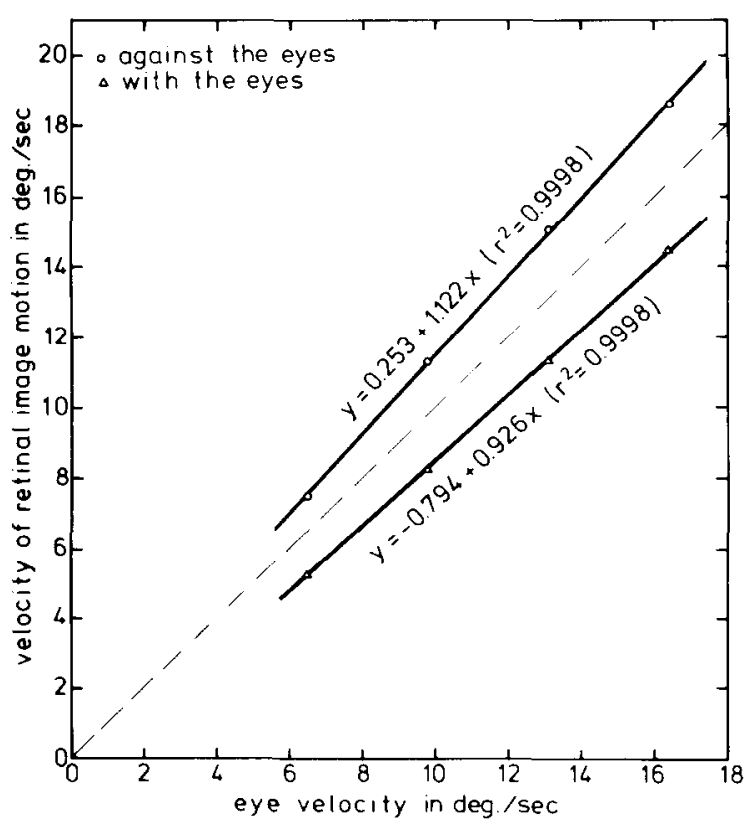

Fig. 3. Velocity of the background image on the retina at threshold as a function of eye movement velocity (solid lines). The broken line represents the points where retinal velocity equals the velocity of the eyes. The JND between the retinal and extraretinal signals is the distance between a point on a solid line and its vertical projection on the broken line.

If one computes for each eye movement velocity the mean of the two corresponding retinal threshold velocities (for the two directional conditions) the linear regression line along these four values almost coincides with the broken line in fig. 3 . (In fact it is slightly, but significantly steeper $-p<0.02-$ the slope being 1.02 .)

\section{Discussion}

During smooth pursuit eye movements, the threshold for motion of a visual stimulus (in this case the background) increases proportionally to ocular velocity (irrespective of whether the stimulus and the eyes move in the same or in the opposite direction). This supports the assumption that the perception of motion depends on the perception of a just noticeable difference between retinal and extraretinal signals. During smooth pursuit retinal and extraretinal signals are compared in terms of velocity. The data show no evidence of main effects of either eye movement amplitude or direction of eye movements relative to the background. The small influences these factors appear to have on the effects of ocular velocity can be neglected. They explain considerably less than $1 \%$ of the variance. 
The broken line in fig. 3 reflects the threshold for motion according to the classical view, which stipulates that no motion is perceived only when retinal and extraretinal signals are equal. The present data indicate that this view is incorrect (at least with respect to smooth pursuit eye movements). There is a whole range (between the two drawn lines) where retinal and extraretinal signals differ, but where no stimulus motion is perceived, and this range becomes larger with faster eye movements.

The range is approximately symmetrical with respect to the points where retinal and extraretinal velocities are equal. If ocular velocity is underrepresented in extraretinal signals, then retinal velocity signals would be equal to extraretinal velocity signals only when the velocity of the eyes is somewhat larger than the velocity of the retinal image shift. The range where no motion is perceived should then be symmetrical to a line with a slope less than 1 (the difference being a measure of the degree of under-registration). Since the observed range is symmetrical to a line with a slope of 1.02 , the underregistration hypothesis must be rejected. If anything, the data indicate a slight over-registration of ocular velocity.

Fig. 3 illustrates that the same retinal image velocity can be interpreted either as movement or as stability of a visual stimulus, depending on the magnitude of the extraretinal velocity signal. Hence the perception of movement in the visual field is relative to the magnitude of the extraretinal signals.

This suggests a new explanation for the so-called Filehne illusion, which is the illusory perception of background motion during ocular pursuit of a stimulus that moves close to the eyes in a plane different from the background (Filehne 1922; Mack and Herman 1973, 1978). When the eyes focus on the nearby stimulus, eye convergence and accommodation are likely to cause the retinal image motions of the background to be displaced slightly from where they would have been if background and stimulus had been in the same plane. Therefore, with respect to the background, retinal and extraretinal signals differ and motion of the background is perceived when the magnitude of this difference reaches the level of the JND. Accordingly, the illusion should be reduced when the stimulus moves at a larger distance from the eyes, or when the distance between the background and the stimulus is reduced and it should disappear completely when they move in the same plane. Also since the JND grows with increasing ocular velocity, the illusion should be reduced with increasing stimulus velocities. Since the illusion is not due to the perception of depth, but is caused by the perception of a difference between retinal and extraretinal signals (relative to the magnitude of the extraretinal signals) it should occur also with monocular viewing. All those effects have indeed been observed (Mack and Herman 1978).

The perceived direction of motion of a visual stimulus is also relative to the magnitude of extraretinal velocity signals. Fig. 3 shows that the same retinal image velocity can be perceived either as movement of the background against 
the direction of the eyes (when the extraretinal signal is smaller) or as background movement in the same direction as the eyes (when the extraretinal signal is larger).

The perceived velocity of a moving stimulus is also relative to the magnitude of the extraretinal signals and appears to be independent of the retinal velocity signal. This should become clear when considering a horizontal cross section of the graph in fig. 3. The effect of an increasing extraretinal signal is first to reduce the perceived velocity of the stimulus, then to maintain it for some time at zero level and finally to increase it again (although in the opposite direction).

The relativity of perceived velocity has an important consequence. Consider fig. 4, where the solid line represents a hypothetical Stevens function obtained when the velocity of a moving background is estimated in the absence of eye movements. The broken lines represent similar functions obtained in the presence of eye movements of two different velocities. These functions are shifted downwards in proportion to the increased threshold for motion of the background (which depends on ocular velocity). Hence the perceived velocity of the background is reduced with faster eye movements. A special case occurs when eye velocity equals the velocity of the background (i.e. when the eyes track the background). Such an instance defines the Aubert-Fleischl phenomenon mentioned earlier: the perceived velocity of a signal pursued with the eyes is lower than when the signal moves past stationary eyes. Since the threshold

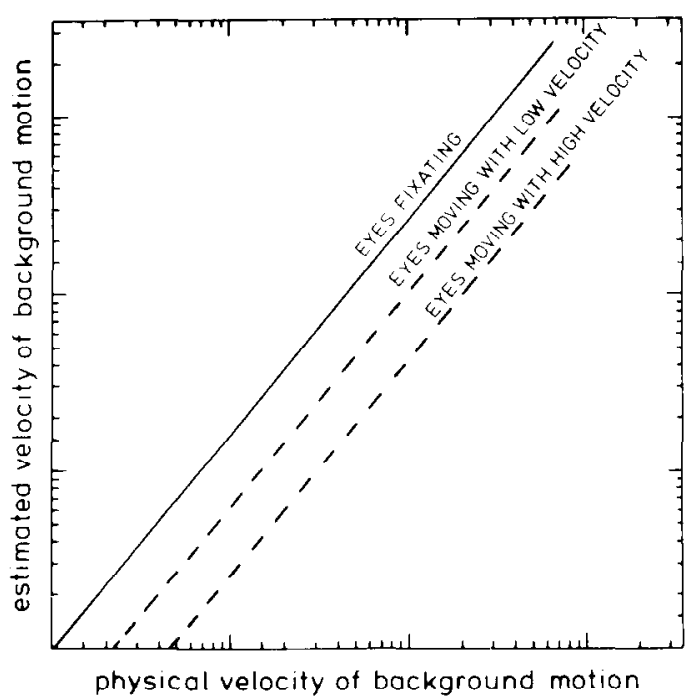

Fig. 4. Theoretical Stevens functions: estimated background velocity as a function of physical background velocity for non-moving eyes (drawn line) or moving eyes (broken lines). 
for motion increases with faster eye movements the Aubert-Fleischl phenomenon should increase with signals moving at higher velocities. This expectation is confirmed in a study on the Aubert-Fleischl phenomenon reported by Dichgans et al. (1975).

These considerations taken together imply that perceived motion, velocity and direction are all relative concepts. They are the outcome of a comparison process. Retinal image shifts do not carry information about movement of a stimulus. To perceive motion a comparison signal is needed. In this sense the perception of motion does not differ from the physical determination of motion of an object. Here too the object itself does not carry information on its own movement. Its motion, velocity and direction can only be determined with respect to another object, serving as a frame of reference. In the absence of additional retinal signals that can serve as a frame of reference [3], the visual system uses extraretinal signals for this purpose.

Since extraretinal signals are so crucial to the perception of motion it is worthwhile to speculate on their nature. Illusory motion and displacement may be perceived with stationary eyes. This is the case when an effort is made to move paralyzed eyes (see Kornmüller 1930; Stevens et al. 1976), or in an attempt to keep the eyes stationary during mechanical loading of the eyeball (Skavensky et al. 1972; Bridgeman 1979). Since in such cases retinal motion signals equal zero, the illusions must have originated from the presence of extraretinal signals concurrent not with eye movements but with the intention to move the eyes.

However, thresholds for motion do not only increase during eye movements. They also increase during active head rotations, both in the presence and absence of eye movements (Wallach and Kravitz 1965; Wallach and Bacon 1976; Büchele et al. 1980; Probst et al. 1980). Exploratory measurements in our own experimental set-up have revealed that the threshold for motion of the background is also elevated when the eyes and head move together (the effects of body rotation are currently under investigation). There is a large amount of literature on visual movement illusions concurrent with bodily motion and acceleration, such as illusory motion of visual afterimages, the oculogravic and the oculogyral illusions, the elevator illusion and many illusory distortions of tilt, size, velocity, direction and distance (see Fisher and Kornmüller 1930; Mack and Bachant 1969, Ross 1974; Pavard and Berthoz 1977; Yasui and Young 1975; Dichgans and Brandt 1978).

These phenomena, taken together, suggest that in response to acceleration, the vestibular system creates a signal which is functionally equivalent (and

[3] The extent to which the visual system selects other retinal signals to serve as a frame of reference seems to depend on their Gestalt properties in the visual field (see for some discussions of this issue and of its relevance to induced motion: Wallach 1959; Ross 1974; Wallach et al. 1978; Epstein 1978; Goodson et al. 1980; Rock et al. 1980). 
probably complementary) to the extraretinal signal associated with the oculomotor system. This equivalence would imply that a JND must be reached also between retinal signals and such vestibular signals in order to perceive visual movement of a stimulus during head or body movement. Hence visual thresholds for motion should also increase in direct proportion to head or body velocity. Recently this effect has indeed been reported (Probst et al. 1980). This "equivalence hypothesis" is also supported by an effect reported by Pavard and Berthoz (1977). Subjects looked at stimuli moving with constant velocity over a screen. When the whole experimental set-up (including the subjects' chair, the screen and the stimulus projection apparatus) was accelerated, subjects perceived a cessation of stimulus motion for a certain period, the duration of which increased with higher accelerations. The effect can be explained if it is assumed that stimulus motion is not perceived when the vestibular velocity signals reach a magnitude which approximates the magnitude of the retinal velocity signals. Since the range over which no motion is perceived should also increase with larger vestibular signals (as depicted in fig. 3) the effect will last longer with higher accelerations.

In conclusion, it seems that the reference signals - without which retinal image motion cannot be interpreted in terms of stimulus movement-do not only concur with the intention to move the eyes, but also result from movements of the body: apparently such reference signals are evoked by what could be termed "ego-motion". Since motion can only be defined by virtue of these reference signals, the perception of a moving or stationary stimulus can be considered as a psychological construct, created in the wake of our actions. The consequences for the epistemology of perception (see Carterette and Freedman 1974; Gibson 1968, 1979; Ullman 1980), at least with respect to movement, are obvious.

\section{References}

Aubert, H., 1886. Die Bewegungsempfindung. Pflügers Archiv 39, 347-370.

Aubert, H., 1887. Die Bewegungsempfindung, Zweite Mitteilung. Pflügers Archiv 40, 459-480.

Beeler, G.W., 1967. Visual threshold changes resulting from spontaneous saccadic eye movements. Vision Research 7, 769-775.

Bridgeman. B., 1979. Adaptation and the two-visual-systems hypothesis. The Behavioral and Brain Sciences 2, 64-65.

Bridgeman, B. and L. Stark, 1979. Omnidirectional increase in threshold for image shifts during saccadic eye movements. Perception \& Psychophysics 25, 241-243.

Bridgeman, B., D. Hendry and L. Stark, 1973. Failure to detect displacement of the visual world during saccadic eye movements. Vision Research 15, 719-722.

Bridgeman, B., S. Lewis, G. Heit and M. Nagle, 1979. Relation between cognitive and motororiented systems of visual position perception. Journal of Experimental Psychology: Human Perception and Performance 5, 692-700.

Büchele. W., D. Degner and Th. Brandt, 1980. Thresholds for object-motion perception raised by 
concurrent head-movements. Proceedings of the 53rd meeting of the German physiological society, March 18-21, 1980. Pflügers Archiv Supp. Vol. 384. R 33, 131.

Carterette, E.C. and M.P. Friedman (eds.), 1974. Handbook of perception. Vol. I: Historical and philosophical roots of perception. New York: Academic Press.

Dichgans, J. and Th. Brandt, 1978. Visual-vestibular interaction: effects on self-motion perception and postural control. In: R. Held, H.W. Leibowitz and H.L. Teuber (Eds.), Handbook of sensory physiology. Vol. VIII: Perception. New York: Springer Verlag.

Dichgans, J. and R. Jung, 1969. 'Attention, eye movements and motion detection: facilitation and selection in optokinetic nystagmus and railway nystagmus'. In: C.R. Evans and T.B. Mulholland (eds.), Attention in neurophysiology. London: Butterworths. pp. 348-376.

Dichgans, J., F. Körner and K. Voigt, 1969. Vergleichende Skalierung des afferenten und efferenten Bewegungssehens beim Menschen: linearen Funktionen mit verschiedenen Austiegssteilkeit. Psychologische Forschung 32, 277-295.

Dichgans, J., E. Wist, H.C. Diener and Th. Brandt, 1975. The Aubert-Fleischl phenomenon: a temporal frequency effect on perceived velocity in afferent motion perception. Experimental Brain Rescarch 23, 529-533.

Epstein, W., 1978. Two factors in the perception of velocity at a distance. Perception \& Psychophysics 24, 105-114.

Filehne. W.. 1922. Über das optische Wahrnehmen von Bewegungen. Zeitschrift für Sinnesphysiologie 53, 134-145.

Findlay, J.M., 1974. Direction perception and human fixation eye movements. Vision Research 14. $703-711$.

Fisher, M.H. and A.E. Kornmüller, 1930. Optokinetisch ausgelöste Bewegungswahrnehmungen und optokinetischer Nystagmus. Journal für Psychologie und Neurologie 41, 273-308.

Fleischl, E. von, 1882. Physiologisch-optische Notizen. Sitzungsbez, Akad. Wissensch. III(86), 7-25. Wien.

Gibson. J.J., 1968. What gives rise to the perception of motion? Psychological Review 75, 335-346.

Gibson, J.J., 1979. The ecological approach to visual perception. Boston: Houghton Miffin.

Goodson, F.E., F.Q. Snider and J.E. Swearingen, 1980. Motion parallax in the perception of movement by a moving subject. Bulletin of the Psychonomic Society 16, 87-88.

Gyr, J.W., 1972. Is a theory of direct visual perception adequate? Psychological Bulletin 77. $246-261$.

Hazelhoff, F. and H. Wiersma, 1925. Die Wahrnehmungszeit. Zeitschrift für Psychologie 97. $174-190$.

Helmholtz, H. von, 1962. Treatise on physiological optics. Vol. III. (Translated from the 3rd German edition 1867; J.P.C. Southall (ed.).) New York: Dover.

Hoist. E. von, 1954. Relations between the central nervous system and the peripheral organs. British Journal of Animal Behaviour 2, 89-94.

Jeannerod, M., H. Kennedy and M. Magnin, 1979. Corollary discharge: its possible implications in visual and oculomotor interactions. Neuropsychologia 17, 241-258.

Kornmöllcr, A.E., 1930. Eine experimentelle Anästhesie der äusseren Augenmuskeln am Menschen und ihre Auswirkungen. Journal für Psychologie und Neurologie 41, 354-366.

Legge, G.E. and F.W. Campbell, 1981. Displacement detection in human vision. Vision Research $21,205-213$.

Mack, A., 1970. An investigation of the relationship between eye and retinal image movement in the perception of movement. Perception \& Psychophysics 8, 291-298.

Mack, A. and J. Bachant, 1969. Perceived movement of the after image during eye movements. Perception \& Psychophysics 6, 379-384.

Mack, A. and E. Herman, 1972. A new illusion: the underestimation of distance during pursuit cyo movements. Perception \& Psychophysics 12, 471-473.

Mack, A. and E. Herman, 1973. Position constancy during pursuit eye movement: an investigation of the Filehne illusion. Quarterly Journal of Experimental Psychology 25, 71-84. 
Mack, A. and E. Herman, 1978. The loss of position constancy during pursuit eye movements. Vision Research 18, 55-62.

MacKay, D.M., 1972. Voluntary eye movements as questions. In: Cerebral control of eye movements and motion perception. Bibl. Ophthal. 82, 369-376.

MacKay, D.M., 1973. 'Visual stability and voluntary eye movement'. In: R. Jung (ed.), Handbook of sensory physiology, Vol. VIl/3. Heidelberg/New York: Springer Verlag.

MacKay, D.M. and H. Mittelstaedt, 1974. Visual stability and motor control (reafference revisited). Cybernetics and Bionics. Munich/Vienna: Oldenburg.

Mateeff, S., 1978. Saccadic eye movements and localization of visual stimuli. Perception \& Psychophysics 24, 215-224.

Matin, E., 1974. Saccadic suppression: a review and an analysis. Psychological Bulletin 81, $899-917$.

Matin, L., 1972. 'Eye movements and perceived visual direction'. In: Hurvich and Jameson (eds.), Handbook of sensory physiology. Vol. VII/4: Visual psychophysies. Heidelberg: Springer Verlag.

Miles, F.A. and E.V. Evarts, 1979. Concepts of motor organization. Annual Review of Psychology $30,327-362$.

Monahan, J.S., 1972. Extraretinal feedback and visual localization. Perception \& Psychophysics 12, 349-353.

Pavard, B. and A. Berthoz, 1977. Linear acceleration modifies the perceived velocity of a moving visual scene. Perception 6, 529-540.

Probst, Th., D. Degner and Th. Brandt, 1980. Object motion perception affected by concurrent self-motion. Proceedings of the 3rd European Conference on Visual Perception, 1980, University of Sussex, Brighton, England. Unpublished manuscript.

Rock, I., M. Auster, M. Schiffman and D. Wheeler, 1980. Induced movement based on subtraction of motion from the inducing object. Journal of Experimental Psychology: Human Perception and Performance 6, 391-403.

Ross, H., 1974. Behaviour and perception in strange environments. London: George Allen and Unwin.

Skavenski. A.A., G. Haddad and R.M. Steinman. 1972. The extraretinal signal for the visual perception of direction. Perception \& Psychophysics 11, 257-290.

Stevens, J.K., R.C. Emerson, G.L. Gerstein, T. Kallos, G.R. Neufeld, C.W. Nichols and A.C. Rosenquist. 1976. Paralysis of the awake human: visual perceptions. Vision Research 16 , 93-98.

Ullman, S., 1980. Against direct perception. The Behavioral and Brain Sciences 3, 373-415.

Wallach, H., 1959. The perception of motion. Scientific American 201, 56-60.

Wallach, H. and J. Bacon, 1976. The constancy of the orientation of the visual field. Perception \& Psychophysies 19, 492-498.

Wallach, H. and H. Kravitz. 1965. The measurement of constancy of visual direction and its adaptation. Psychonomic Science 2, 217-218.

Wallach. H., J. Bacon and P. Schulman, 1978. Adaptation in motion perception: alteration of induced motion. Perception \& Psychophysics 24. 509-514.

Ward, F., 1976. 'Pursuit eye movements and visual localization'. In: R.A. Monty and J.W. Senders (eds.). Eye movements and psychological processes. Hillsdale, NJ: Lawrence Erlbaum. pp. 289-297.

Wertheim, A.H., 1979. Information processed in ocular pursuit. Monograph of the Institute for Perception TNO, Soesterberg. The Netherlands (Ph.D. Thesis).

Wertheim, A.H., 1980. 'Information processing mechanisms involved in ocular pursuit'. In: G.E. Stelmach and J. Requin (eds.), Tutorials in motor behavior. New York: North-Holland.

Whipple, W.R. and H. Wallach, 1978. Direction-specific motion thresholds for abnormal image shifts during saccadic eye movement. Perception \& Psychophysics 24. 349-355.

Yasui, S. and L.R. Young, 1975. Perceived visual motion as effective stimulus to pursuit eye movement system. Science 190, 906-908. 